\title{
IMPACT OF ARTIFICIAL INTELLIGENCE CHANGING THE WORLD
}

\author{
Divya Khare \\ Student CS \& IT, \\ Kalinga university, Raipur
}

\begin{abstract}
The world of artificial intelligence is progressing in a more advanced way these days. In this $21^{\text {st }}$ century of technologies and gadgets all over the world, the devices and systems are designed to do small tasks for instance virtual assistant, facial recognition, and some more minor works. However, the future of $\mathrm{AI}$ is to accomplish tasks in a better and advanced version as humans do. In the long term, an AI system which can do all the day to day general activities from controlling cars to handling advance computerized business system will bring a better change in the world. Humans are still on the verge to create history to create an invention of $\mathrm{AI}$ that could be controlled in every perspective so that human civilization is not harmed and at the same time is advanced to face future challenges. [Ref.1,2] et al. $(2017,2018)$
\end{abstract}

\section{INTRODUCTION}

Artificial Intelligence, the term describes the technology as an artificial brain. It is simply the mixture of machine and advanced programming that is built to learn from experiences on its own and can adapt new pieces of information. [Ref.12]et al.(2020)

"The development of full artificial intelligence could spell the end of the human race....It would take off on its own, and re-design itself at an ever-increasing rate. Humans, who are limited by slow biological evolution, couldn't compete, and would be superseded."- Stephen Hawking. [Ref.03] et al. (2018)

\section{STAGES AND TYPES OF AI}

\section{A. Artificial Narrow Intelligence (ANI)}

This AI can only do the things which are programmed in a system which is the update of $\mathrm{AI}$ in our world to date. [Ref.1, $4,5]$ et al. $(2018,2019,2020)$

\section{B. Artificial General Intelligence (AGI)}

This AI can develop its thinking capability and can learn to do multiple functions as much as a human being does. [Ref.1, 4, 5] et al. $(2018,2019,2020)$
Rahul Chawda HOD CS \& IT, Kalinga University Raipur

\section{Artificial Super Intelligence (ASI)}

This AI will have the ability to surpass all the activities and mental capabilities that a human being has currently. If this stage is brought in reality miracle will happen in the world of technology. [Ref.1, 4, 5] et al. $(2018,2019,2020)$

According to what an AI can function these are the types-

\section{A. Reactive Machines}

This type of AI works only with the present data with some combination of inputs and does not tend to learn new actions. The best example of this is IBM's Deep Blue. [Ref.1, 4, 5] et al. $(2018,2019,2020)$

\section{B. Limited Memory Machines}

This type of $\mathrm{AI}$ is capable of learning from past experiences to evaluate and decide the better future with its temporary memory. The best example of this is Self Driving Cars. [Ref.1, 4, 5] et al. $(2018,2019,2020)$

\section{Theory of Mind}

This type of AI is more of emotional intelligence to learn and act from the human emotional behaviour and needs. This concept is not physically designed and researchers are innovating in this field. [Ref.1, 4, 5] et al. $(2018,2019,2020)$

\section{Self Aware is still}

This type of AI is an imaginary world for the current situation where a machine can be self-aware of what's best for them which can cause harm to the human civilization or can be called the end of human existence. [Ref.1, 4, 5] et al. (2018, 2019, 2020)

\section{TECHNOLOGIES AND TOOLS USED IN AI}

- Natural Language Processing - It enables a machine to understand and interact with a human's natural language. [Ref. 9, 10, 11] et al. $(2017,2020)$

- Speech Recognition - It can translate human speech into codes understandable by a computer system. [Ref. $9,10,11]$ et al. $(2017,2020)$

- Vision Recognition - It allows a system to understand images, structures, shapes to match or compare with the input. [Ref. 9, 10, 11] et al. $(2017,2020)$

- Virtual Agent - It allows a system to process a piece of instructions to assist in their work. [Ref. 9, 10,11] et al. $(2017,2020)$

- Robotics - It allows a robot to be intelligent to learn from the mistakes and perform better in future actions. 


\section{International Journal of Engineering Applied Sciences and Technology, 2020 Vol. 5, Issue 2, ISSN No. 2455-2143, Pages 346-348 \\ Published Online June 2020 in IJEAST (http://www.ijeast.com)}

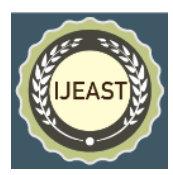

[Ref. 9, 10, 11] et al. $(2017,2020)$

- Gaming - AI can play challenging games as can process any number of possibilities a single turn which can take place like chess. [Ref. 9, 10, 11] et al. $(2017,2020)$

- Biometrics - To identify every single human on the behaviour or physical aspect that makes them unique. [Ref. 9, 10, 11] et al. $(2017,2020)$

- Machine Learning - A field which enables the system to analyze the work done to learn and improve machine on its own. [Ref. 9, 10, 11] et al. $(2017,2020)$

\section{RISKS AND BENEFITS OF AI}

The benefits of AI can make a revolutionary change in our technology. Here are some of the benefits-

An AI can work $24 \times 7$ in a machine resulting in greater efficiency.

- It can do work more accurately than humans giving no errors in results. [Ref. 6, 7] et al. $(2017,2019)$

- AI has simplified our day to day activity a bit as people are using Siri, Alexa, Cortona, and Ok Google to do some of the basic actions. [Ref. 6, 7]

et al. $(2017,2019)$

- AI using the machine as its body can take much more risks than a human can. As we still have not explored many dangerous regions of the earth, for instance deep down in oceans a machine AI can easily explore in places like this. [Ref. 6, 7] et al. $(2017,2019)$

- AI having the capability to learn can solve equations and problems that have not been solved yet by humans providing us discoveries and inventions. [Ref. 6,7] et al. $(2017,2019)$

The dark part of AI comes to the picture now. The risks of AI are -

- Creating an $\mathrm{AI}$ requires high cost and accessing an $\mathrm{AI}$ do the same. [Ref. 6, 7] et al. (2017,2019)

- The machine will take many people's jobs making peoples unemployed in many numbers. [Ref. 6, 7] et al. $(2017,2019)$

- To date, machines are designed just to perform actions according to the data programmed in it. So it cannot take action beyond what's fed. [Ref. 6, 7] et al. $(2017,2019)$

- It will make humans lazy as they will do the major works a human does daily. [Ref. 6, 7] et al. $(2017,2019)$

- A major threat to human civilization, if a Super AI will be created. [Ref. 6, 7] et al. $(2017,2019)$

\section{HOW WILL AI CHANGE THE WORLD?}

Every aspect can be seen to have positive as well as negative effects worldwide.
If we look into the way AI can change the world shortly is like we see in movies with lots of machines all-around humans helping us as well as providing the best result for an input. AI will change the perspective of many major technologies that we see today.

Let's take a look in the future with AI.

- Transportation will have a great impact on AI with self-driving cars combined with a virtual assistant, a total automatic facility in the vehicles around us. [Ref. $1,8]$ et al. $(2017,2018)$

- A machine with AI for our better health that can scan a body and provide the best treatment according to the disease one has or the best medicines for that patient.

[Ref. 1, 8] et al. $(2017,2018)$

- In the world of cyber security where securing data from hackers is the most important concern for a firm. An AI can create a better environment or system to secure data that will always be one step ahead of the hacker's mind. [Ref. 1, 8] et al. $(2017,2018)$

- In the world of public security, facial and voice recognition from CCTV cameras and more developed systems to catch criminals will be easier. [Ref. 1,8$]$ et al. $(2017,2018)$

- With the help of AI, humanoid robots can be made intelligent. So they can work without being preprogrammed. [Ref. 1, 8] et al. $(2017,2018)$

- In agriculture, AI robots can analyze crop growth \& productivity before harvesting according to the weather daily. It can decrease loss to the farmer at its best. [Ref. 1, 8] et al. $(2017,2018)$

\section{CONCLUSION}

With the world changing and adapting AI so fast, we will soon be surrounded by AI and its robotic technology.

However, there is a vast area for us as humans to evolve around the machines and learn from them and vice versa.

Gradually, we will achieve the technology but not to forget, humans need to be ready for every bad circumstance that can occur due to the growth in AI. I think humans should build an AI up to the level they can control and understand their programs and mind. At some point, if they learn or surpass our thinking or understanding capability human race will be in great danger. Let's see what AI has kept for us in the future technological world.

\section{REFERENCES}

[1] Heath Nick (2018), Managing AI and ML in the Enterprise (How will AI change the world?), website (https://www.zdnet.com/article/)

[2] Poola Indrasen (2017), How Artificial Intelligence in Impacting Real life Everyday, website (https://www.researchgate.net/publication/)

[3] Gall Richard (2018), 5 polarising quotes from Professor Stephen Hawking on artificial intelligence (Quote no. 1) 
[4] Lateef Zulaikha (last updated in 2020), Types of Artificial Intelligence you should know, edureka blog(https://www.edureka.co/blog/)

[5] Joshi Naveen (2019), 7 Types of Artificial Intelligence, website (https://www.forbes.com/sites/)

[6] Kumar Sunil (2019), Advantages and Disadvantages of Artificial Intelligence, website (https://towardsdatascience.com)

[7] Abhiindia (2017), Top Benefits and Risks of Artificial Intelligence, website(https://www.studytonight.com/post/)

[8] Gammon Katherine (2017), 5 ways Artificial Intelligence will change the world by 2050, website(https://news.usc.edu/trojan-family/)

[9] Admin Valluri (2017), Artificial Intelligence and its applications,

website(https://www.valluriorg.com/blog/)

[10] Admin javatpoint(last updated in 2020), Application of AI, website(https://www.javatpoint.com)

[11] Admin tutorialspoint(last updated in 2020), Artificial Intelligence - Overview (Application of AI), website(https://www.tutorialspoint.com)

[12] Admin Technopedia,(Last updated on 2020)Artificial Intelligence(AI), Definition, website(https://www.techopedia.com) 